Accepted by ApJ: January 22, 2015

\title{
Resolved Millimeter Emission from the HD 15115 Debris Disk
}

\author{
Meredith A. MacGregor ${ }^{1}$, David J. Wilner ${ }^{1}$, Sean M. Andrews ${ }^{1}$, A. Meredith Hughes ${ }^{2}$
}

\begin{abstract}
We have used the Submillimeter Array (SMA) to make 1.3 millimeter observations of the debris disk surrounding HD 15115, an F-type star with a putative membership in the $\beta$ Pictoris moving group. This nearly edge-on debris disk shows an extreme asymmetry in optical scattered light, with an extent almost two times larger to the west of the star than to the east (originally dubbed the "Blue Needle"). The SMA observations reveal resolved emission that we model as a circumstellar belt of thermal dust emission. This belt extends to a radius of $\sim 110$ AU, coincident with the break in the scattered light profile convincingly seen on the western side of the disk. This outer edge location is consistent with the presence of an underlying population of dust-producing planetesimals undergoing a collisional cascade, as hypothesized in "birth ring" theory. In addition, the millimeter emission shows $\mathrm{a} \sim 3 \sigma$ feature aligned with the asymmetric western extension of the scattered light disk. If this millimeter extension is real, then mechanisms for asymmetry that affect only small grains, such as interactions with interstellar gas, are disfavored. This tentative feature might be explained by secular perturbations to grain orbits introduced by neutral gas drag, as previously invoked to explain asymmetric morphologies of other, similar debris disks.
\end{abstract}

Subject headings: circumstellar matter — stars: individual (HD 15115) — submillimeter: planetary systems

\section{Introduction}

Nearly a hundred dusty debris disks around nearby stars have been spatially resolved at one or more wavelengths (Matthews et al. 2014). The bulk radial structure of these

\footnotetext{
${ }^{1}$ Harvard-Smithsonian Center for Astrophysics, 60 Garden Street, Cambridge, MA 02138, USA

${ }^{2}$ Department of Astronomy, Van Vleck Observatory, Wesleyan University, 96 Foss Hill Drive, Middletown, CT 06459, USA)
} 
disks is generally well explained by the presence of a localized belt of planetesimals, or "birth ring," where smaller and smaller dust grains are produced in a catastrophic collisional cascade and dispersed (Strubbe \& Chiang 2006; Augereau \& Beust 2006). However, many disks exhibit additional substructure such as brightness asymmetries, offsets, warps, and clumps that cannot be explained by the steady-state collisional models assumed in this framework. Numerous mechanisms have been proposed to explain morphologies that depart from axial symmetry, including interactions with interstellar gas, planetary resonances, and stellar flybys (Debes et al. 2009; Maness et al. 2009, 2008; Kalas et al. 2007, and references therein). Still, most of these structures have been detected only at optical and infrared wavelengths, probing a population of small grains that react strongly to stellar radiation and winds. Additional observational constraints, particularly at millimeter wavelengths, are needed to access larger grains with dynamics more similar to the parent colliding bodies, to solidify our understanding of the mechanisms that shape debris disk structure.

HD 15115 is an F2V star at $45 \pm 1$ pc (van Leeuwen 2007) whose space motions suggest membership in the young $\beta$ Pictoris moving group (Moór et al. 2011), which includes the well-studied $\beta$ Pic and AU Mic debris disks. Although the case for membership in this group is not ironclad (see Debes et al. 2008), recent estimates suggest an age of $21 \pm 4$ Myr for these stars (Binks \& Jeffries 2014). An infrared excess from HD 15115 indicating orbiting dust was first noted from IRAS observations (Silverstone 2000). Subsequent scattered light imaging from HST, Keck, LBT, and Gemini have resolved a remarkable edge-on circumstellar disk (Kalas et al. 2007; Debes et al. 2008; Rodigas et al. 2012; Schneider et al. 2014; Mazover et al. 2014). This disk shows an extreme asymmetry in optical scattered light (Kalas et al. 2007): the east side of the disk extends to $\sim 7^{\prime \prime}$ (315 AU), while the west side reaches $>12^{\prime \prime}(>550 \mathrm{AU})$. Moreover, there are indications that this asymmetry has roots at smaller scales. As described by Debes et al. (2008), the optical surface brightness drops steadily on the east side from $\sim 1^{\prime \prime}(<45 \mathrm{AU})$, while the western side appears to flatten interior to $\sim 2^{\prime \prime}(\sim 90 \mathrm{AU})$. In "birth ring" theory, this flattening corresponds to the outer edge of the planetesimal belt where smaller grains are created in collisions and are subsequently launched by stellar forces into wider bound and unbound orbits. The presence of a ring structure is supported by a subtle concavity visible in scattered light to the north of the star (Schneider et al. 2014), and this brighter side of a ring becomes clear in recent high contrast images (Mazoyer et al. 2014).

A $3 \sigma$ detection of $850 \mu \mathrm{m}$ continuum emission from the HD 15115 system using the James Clerk Maxwell Telescope/SCUBA suggested the presence of a reservoir of large dust grains in the disk, with low temperature $\left(T_{d}=62 \mathrm{~K}\right)$ and low mass $\left(\sim 0.047 \mathrm{M}_{\oplus}\right)$ indicative of debris (Williams \& Andrews 2006). Subsequent mapping observations with SCUBA-2 obtained a consistent but higher flux density $(8.5 \pm 1.2 \mathrm{mJy}$ vs. $4.9 \pm 1.6 \mathrm{mJy})$ and did 
not resolve any structure with a 14" (FWHM) beam (Panić et al. 2013). In light of the asymmetric morphology of the HD 15115 disk seen in scattered light, we have used the Submillimeter Array (SMA) 11 to resolve the millimeter emission from the disk, aimed at tracing the distribution of underlying planetesimals. These observations reveal a planetesimal belt together with tentative evidence for an asymmetric extension of millimeter emission to the west, coincident with the extended feature seen in optical scattered light. We quantify the detected millimeter structure and discuss the plausibility of proposed mechanisms for the asymmetry.

\section{Observations}

We observed HD 15115 in fall 2013 with the SMA (Ho et al. 2004) on Mauna Kea, Hawaii at a wavelength of $1.3 \mathrm{~mm}$ using both the compact and extended configurations of the array. Table 1 summarizes the essentials of these observations, including the dates, baseline lengths, and atmospheric opacity. With the extended baselines, these observations probe angular scales down to $\lesssim 1^{\prime \prime}$. While typically only six (or fewer) of the eight array antennas were available during the five tracks, the weather conditions were generally very good for observations at this wavelength. The total bandwidth available was $8 \mathrm{GHz}$ derived from two sidebands spanning \pm 4 to $8 \mathrm{GHz}$ from the local oscillator (LO) frequency. The phase center was located at $\alpha=02^{\mathrm{h}} 26^{\mathrm{m}} 16^{\mathrm{s}} .24, \delta=+06^{\circ} 17^{\prime} 33^{\prime \prime} 19$ (J2000), corresponding to the position of the star uncorrected for its proper motion of $(86.31,-49.97){\text { mas } \mathrm{yr}^{-1}}^{-1}$ (van Leeuwen 2007). The $\sim 54^{\prime \prime}$ (FWHM) field of view is set by the primary beam size of the 6 - $\mathrm{m}$ diameter array antennas.

The data from each track were calibrated independently using the IDL-based MIR software package. Time-dependent complex gains were determined using observations of two nearby quasars, J0224+069 (0.8 away) and J0238+166 (10.8 away), interleaved with observations of HD 15115 in a 12 minute cycle for the extended tracks and a 16 minute cycle for the compact tracks. The passband shape was calibrated using available bright sources, mainly 3C84 or 3C454.3. Observations of Uranus during each track were used to derive the absolute flux scale with an estimated accuracy of $\sim 10 \%$. Imaging and deconvolution were performed with standard routines in the MIRIAD software package. A variety of visibility weighting schemes were used to explore compromises in imaging between higher angular

\footnotetext{
${ }^{1}$ The Submillimeter Array is a joint project between the Smithsonian Astrophysical Observatory and the Academica Sinica Institue of Astronomy and Astrophysics and is funded by the Smithsonian Institution and the Academica Sinica.
} 
resolution and better surface brightness sensitivity.

\section{Results and Analysis}

\section{1. $\quad 1.3 \mathrm{~mm}$ Dust Continuum Emission}

Figure 1 shows a contour image of the 1.3 millimeter emission overlaid on a Hubble Space Telescope/Advanced Camera for Surveys coronographic image of optical scattered light (F606W filter) from Kalas et al. (2007). The synthesized beam size for this 1.3 millimeter image, obtained with natural weighting, is $3^{\prime \prime} .0 \times 2^{\prime \prime} .1(135 \times 95 \mathrm{AU})$, position angle $73^{\circ}$. The rms noise is $0.30 \mathrm{mJy}_{\text {beam }}{ }^{-1}$, and the peak signal-to-noise ratio is about 5 . The red star symbol indicates the stellar position, corrected for proper motion, i.e. offset from the phase center by $\left(1^{\prime \prime} .3,-0^{\prime \prime} \cdot 7\right)$. This position coincides very closely with the emission peak, well within the combined uncertainties of the absolute astrometry and modest signal-to-noise. The image reveals a narrow band of 1.3 millimeter continuum emission that extends from the east-southeast to west-northwest, aligned closely with the optical scattered light disk orientation. The western extent of the millimeter emission appears to be greater than the eastern extent, although the significance of this elongation is not high.

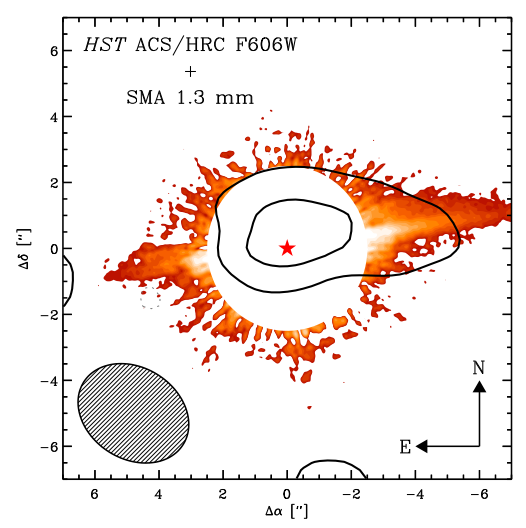

Fig. 1. - SMA image of the 1.3 millimeter continuum emission from HD 15115, overlaid on an image of optical scattered light from the Hubble Space Telescope (Kalas et al. 2007). The contour levels are in steps of $2 \times 0.3 \mathrm{mJy}$, the rms noise level. The ellipse in the lower left corner indicates the $3^{\prime \prime} .0 \times 2$.' $1(\mathrm{FWHM})$ synthesized beam size. The star symbol marks the position of the stellar photosphere, corrected for proper motion. 


\subsection{Disk Modeling}

In order to characterize the millimeter emission from HD 15115, we used the procedure described by MacGregor et al. (2013) that employs Markov Chain Monte Carlo (MCMC) methods to fit a simple parametric model to the observed visibilities. We assume that the emission arises from a geometrically thin, axisymmetric belt characterized by a surface brightness profile of the form $I_{\nu}(r) \propto r^{x}$ for $R_{\text {in }}<r<R_{\text {out }}$. The belt emission normalization is defined by a total flux density $F_{\text {belt }}=\int I_{\nu} d \Omega$, and the belt center is given by offsets relative to the pointing center $\{\Delta \alpha, \Delta \delta\}$. The various scattered light observations show that the disk is nearly edge-on to the line of sight. For our models, we adopt an inclination angle of $87^{\circ}$ and an orientation on the sky described by a position angle of $278.5 \pm 0.5$ (Kalas et al. 2007). Small variations in these values have no material impact on the results. This initial model does not attempt to account for any asymmetric structure in the emission. To address the western extension, we also made models that included an unresolved source with flux density $F_{\text {res }}$ and position $\Delta r$, defined as an offset from the belt center along the position angle of the disk major axis.

For a given parameter set, we compute synthetic visibilities corresponding to the SMA observations and compare directly to the data with a $\chi^{2}$ value (the sum of real and imaginary components over all spatial frequencies). By fitting the visibility data directly, we are not sensitive to the non-linear effects of deconvolution, and take full advantage of the complete range of spatial frequencies sampled by the observations. We assumed uniform priors for all parameters, with reasonable bounds imposed to ensure that the model was well-defined: $F_{\text {belt }} \geq 0$ and $0 \leq R_{\text {in }}<R_{\text {out }}$. In addition, we constrained the two parameters $\{\Delta \alpha, \Delta \delta\}$ that describe the belt center to be within 0 .'5 of the offsets predicted from the stellar proper motion; this generously accomodates the uncertainties in the proper motion and the absolute astrometry of the observations. The fit quality is characterized by a likelihood metric, $\mathcal{L}$, determined from the $\chi^{2}$ values $\left(\ln \mathcal{L}=-\chi^{2} / 2\right)$. We make use of the affine-invariant ensemble MCMC sampler proposed by Goodman \& Weare (2010) and implemented effectively in

Python by Foreman-Mackey et al. (2013). With this MCMC approach, we can characterize efficiently the multidimensional parameter space of this simple model and determine posterior probability distribution functions for each parameter by marginalizing over all other parameters in turn.

\subsection{Results of Model Fits}

Figure 2 shows the output of $\sim 10^{4}$ MCMC trials and Table 2 lists the best-fit model parameter values and their $68 \%$ uncertainties determined from the marginalized posterior 
probability distributions for the full model (axisymmetric belt plus western extension). Figure 3 shows comparisons between the data and the best-fit models in the image plane, including the imaged residuals in the rightmost panels. The simple symmetric disk model in the upper panels of Figure 3 reproduces the bulk of the millimeter emission well, but the residual image shows $\mathrm{a} \sim 3 \sigma$ feature coincident with the western extension of the scattered light along the disk axis, and this model yielded a reduced $\chi^{2}=3.67$ (153,032 independent data points, 6 free parameters). The departure from unity reflects the poor fit of the model, in part due to the presence of the residual western emission. By including additional parameters in the model that account for this residual feature $\left(F_{\text {res }}\right.$ and $\left.\Delta r\right)$, the model fit was more satisfactory, as can be seen in the lower panels of Figure 3, with reduced $\chi^{2}=1.88$.

The total flux density of the best fit model is constrained to be $F_{\text {belt }}=2.6_{-0.8}^{+0.5} \mathrm{mJy}$. If we extrapolate the available submillimeter measurements in the literature using the typical spectral index of $\sim 2.65$ for debris disks at these wavelengths (Gáspár et al. 2012), we find close agreement with the SMA value. In particular, extrapolating the SCUBA-2 observation of Panić et al. (2013) to $1.3 \mathrm{~mm}$ gives $2.8 \pm 0.4 \mathrm{mJy}$, while the older SCUBA observation of Williams \& Andrews (2006) gives $1.6 \pm 0.5 \mathrm{mJy}$. Given this consistency, it seems likely that the SMA observations detect the full disk emission and do not miss any significant, more extended, millimeter flux.

The location of the outer edge of the modelled millimeter emission belt, $R_{\text {out }}=110_{-22}^{+31} \mathrm{AU}$, can be compared to expectations based on the scattered light radial surface brightness profile and "birth ring" theory. The HST imaging of HD 15115 by Debes et al. (2008) shows that the scattered light profile steepens on the western side of the disk, falling as $r^{-3.56 \pm 0.06}$ beyond 1".8 (81 AU). The ground based scattered light observations of Rodigas et al. (2012) show a similar break in the western surface brightness profile near $\sim 1.8-2^{\prime \prime}(\sim 80-90$ $\mathrm{AU})$. The model fit to the outer edge of the millimeter belt is consistent with both of these determinations, within the substantial uncertainties. However, in the context of theory, it remains unclear why the eastern side of the scattered light disk shows no clear break in surface brightness slope.

The observations of Rodigas et al. (2012) additionally show a drop in the scattered light surface brightness of the disk's western side interior to $1^{\prime \prime}$ (45 AU), presumably marking the inner edge of the belt. The higher contrast images of Schneider et al. (2014) and Mazoyer et al. (2014) confirm this feature and suggest at least a partial clearing within this radius. Analysis of the spectral energy distribution of the system by Moór et al. (2011) yields a best-fit model with an inner warm component and an outer cold ring at a minimum radius of $42 \pm 2 \mathrm{AU}$. Both of these determinations of the inner disk edge match the (poorly constrained) best-fit inner radius of the millimeter belt, $R_{\text {in }}=43 \pm 28 \mathrm{AU}$. 
Despite the modest signal-to-noise ratio of the millimeter observations, this model fitting exercise yields constraints on the inner and outer radii of the millimeter emission belt that are consistent with previous observations and compatible with the "birth ring" model of debris disks.

Not surprisingly, given the limits of the resolution and sensitivity of the SMA data, model fitting does not provide a strong constraint on the power-law gradient of the millimeter radial emission profile, $x=-0.75_{-0.87}^{+1.37}$. The well known degeneracy between the emission gradient, $x$, and disk outer radius, $R_{\text {out }}$, (e.g. Mundy et al. 1996) is apparent in Figure 2 as a slope introduced in the lower contours resulting from that pair of model parameters. For very negative values of $x$, the contours spread to span a wide range of potential outer radii, as the width and extent of the emission belt become difficult to constrain due to the low brightness in the outer regions. Similarly, the inner radius of the disk is poorly constrained for positive gradients. If we assume that the emitting dust is in radiative equilibrium with stellar heating, leading to a temperature gradient described by $T \propto r^{-0.5}$, then taking the best fit exponent at face value implies a relatively shallow surface density profile, $\Sigma \propto r^{-0.25}$ (albeit with large uncertainty).

\section{Discussion}

Millimeter imaging of HD 15115, like its suspected sister stars in the $\beta$ Pictoris moving group, $\beta$ Pic (Wilner et al. 2011) and AU Mic (Wilner et al. 2012; MacGregor et al. 2013), shows a resolved belt of emission with outer edge coincident with a previously observed break in scattered light, consistent with an underlying "birth ring" of colliding planetesimals. For all of these stars, resolved multi-wavelength datasets suggest planetesimal collisions within a belt produce grains with a spectrum of sizes, and the effects of size-dependent dust dynamics produce the compact millimeter emission and an extended scattered light halo. HD 181327, a fourth member of the $\beta$ Pictoris moving group, also shows a belt of cold dust seen in Herschel PACS $70 \mu \mathrm{m}$ and ATCA $3.2 \mathrm{~mm}$ images (Lebreton et al. 2012), though higher resolution millimeter images are still needed to determine its underlying structure.

The detection of $\mathrm{a} \sim 3 \sigma$ residual along the western extent of the HD 15115 disk, aligned with the asymmetry seen in scattered light from small grains, provides tantalizing evidence that the distribution of larger grains in this system may be asymmetric as well. Similar asymmetric debris disk morphologies in scattered light have been observed in other highly inclined systems where the geometry favors detection, notably HD 32297 (Currie et al. 2012; Debes et al. 2009; Boccaletti et al. 2012) and HD 61005 (Hines et al. 2007; Maness et al. 2009), as well as $\beta$ Pic (Kalas \& Jewitt 1995). While all of these disks show unique mor- 
phological details, similar physical mechanisms may be responsible for the overall shaping of the asymmetric appearance of the debris. However, it is unclear if the asymmetries can be clearly traced back to the dust-producing parent bodies. The constraints from millimeter observations so far fail to provide a consistent picture. HD 32297 was observed at 1.3 millimeters in the early days of CARMA (Maness et al. 2008), and the millimeter image shows a hint of asymmetry, albeit at low signal-to-noise. On the other hand, SMA 1.3 millimeter observations of HD 61005 (Ricarte et al. 2013) provide no evidence that the millimeter grains are present in the spectacular swept-back wings seen in scattered light.

The most common mechanisms invoked to explain the scattered light asymmetries in these highly inclined debris disks involve interactions with the local interstellar medium (ISM). Ram pressure from interstellar gas can remove bound and unbound grains from a disk directly, or by inducing outflows of disk gas that entrain the grains (Maness et al. 2009; Debes et al. 2009). Additionally, neutral gas can introduce secular perturbations to the orbits of bound grains, producing significant effects on disk morphology on orbital timescales (Maness et al. 2009). Alternatively planets within the system can have dust-trapping resonances that create clumpy, wavelength-dependent grain distributions (Wyatt 2006). Looking outside of the system entirely, external perturbation by a close stellar flyby can also shape the distribution of disk grains (Kalas et al. 2007). An increase in the dust grain scattering cross section or a local density enhancement could produce a local increase of collisions (Mazoyer et al. 2014). Finally, azimuthal asymmetries could be generated through the "photoelectric instability" effect, if the disk has a high enough total dust to gas mass ratio (Lyra \& Kuchner 2013). Each of these mechanisms affects different sized grains preferentially and works under specific physical conditions. In the following, we examine the plausibility of each of these mechanisms for creating asymmetric structures in the HD 15115 disk and similar systems.

\subsection{Interactions with the ISM}

Because the proper motion of HD 15115 is nearly parallel to the disk major axis, Debes et al. (2009) suggest that the dominant disk asymmetry in scattered light arises from an ongoing interaction with the local ISM. In this picture, the eastern side of the disk plows head first into the ISM, causing that side to become truncated, while small grains are blown out to the west. Motivated by the swept back morphologies of HD 32297, HD 15115, and HD 61005, Debes et al. (2009) construct a model for a disk interacting with the gaseous ISM, based on the gas drag felt by meteorites entering the Earth's atmosphere. This results in a scaling law for the radial distance $\left(R_{\text {deflect }}\right)$ at which unbound grains are significantly 
perturbed from their original circumstellar orbits:

$$
R_{\text {deflect }}=286\left(\frac{1.67 \times 10^{-22} \mathrm{~g} \mathrm{~cm}^{-3}}{\rho_{\text {gas }}}\right)^{\frac{1}{2}}\left(\frac{20 \mathrm{~km} \mathrm{~s}^{-1}}{v_{\text {rel }}}\right)\left(\frac{L_{\star}}{L_{\odot}}\right)^{\frac{1}{2}} \mathrm{AU},
$$

where $\rho_{\text {gas }}$ is the ISM gas density and $v_{\text {rel }}$ is the relative cloud-disk velocity. By applying the definition of $\beta$, the ratio of the force of radiation pressure to the gravitational force exerted by the star, Equation (1) can be recast into a scaling relation for the ISM gas density $\left(n_{\text {gas }}\right)$ and disk relative velocity $\left(v_{\text {rel }}\right)$ needed to perturb unbound grains from their orbits. Assuming perfectly absorbing spherical grains (Burns et al. 1979)

$$
\beta=0.574\left(\frac{L_{\star}}{L_{\odot}}\right)\left(\frac{M_{\star}}{M_{\odot}}\right)^{-1}\left(\frac{1 \mathrm{~g} \mathrm{~cm}^{-3}}{\rho_{\text {dust }}}\right)\left(\frac{1 \mu \mathrm{m}}{r_{\text {dust }}}\right),
$$

which yields the relation

$$
\begin{aligned}
\left(\frac{n_{\text {gas }}}{200 \mathrm{~cm}^{-3}}\right)\left(\frac{v_{\text {rel }}}{30 \mathrm{~km} \mathrm{~s}^{-1}}\right)^{2} \gtrsim & 0.6262\left(\frac{M_{\star}}{0.95 M_{\odot}}\right)\left(\frac{a_{\text {dust }}}{0.1 \mu \mathrm{m}}\right) \\
& \times\left(\frac{\rho_{\text {dust }}}{2 \mathrm{~g} \mathrm{~cm}^{-3}}\right)\left(\frac{70 \mathrm{AU}}{r}\right)^{2} .
\end{aligned}
$$

Interpreting HST images of HD 61005, Maness et al. (2009) discuss a second model for ISM interaction in which an interstellar gas cloud removes both bound and unbound grains from a disk due to ram pressure stripping. In this scenario, the drag force on a grain must be comparable to or exceed the gravitational force binding the grain to the star. For the bound case, Maness et al. (2009) show that this leads to the following scaling relation for the cloud density ( $\left.n_{\text {gas }}\right)$ and the relative cloud-disk velocity (again $v_{\text {rel }}$ )

$$
\begin{aligned}
\left(\frac{n_{\text {gas }}}{200 \mathrm{~cm}^{-3}}\right)\left(\frac{v_{\text {rel }}}{30 \mathrm{~km} \mathrm{~s}^{-1}}\right)^{2} \gtrsim & \left(\frac{M_{\star}}{0.95 M_{\odot}}\right)\left(\frac{a_{\text {dust }}}{0.1 \mu \mathrm{m}}\right) \\
& \times\left(\frac{\rho_{\text {dust }}}{2 \mathrm{~g} \mathrm{~cm}^{-3}}\right)\left(\frac{70 \mathrm{AU}}{r}\right)^{2} .
\end{aligned}
$$

A similar relation is obtained for the unbound case with an additional constant factor accounting for the relative distance traveled by a grain parallel and perpendicular to the disk 
midplane. Although formulated somewhat differently from Debes et al. (2009), the resulting expression is functionally identical with the exception of a constant factor (compare equations 3 and 4).

While ram pressure stripping of disk grains by the ISM seems like an attractive explanation for the asymmetry seen in systems like HD 15115, Marzari \& Thébault (2011) use numerical modeling to show that even grains just above the disk blow-out size $(1-10 \mu \mathrm{m})$ are minimally affected by ISM interactions and are lost from the disk before they acquire any significant inclination. The millimeter grains, with much smaller values of $\beta$, should feel little to no effect from ISM gas. Thus, this argument has difficulty explaining an asymmetry in the millimeter disk emission. Moreover, Maness et al. (2009) point out that the ISM densities required are characteristic of cold clouds $\left(n \sim 50 \mathrm{~cm}^{-3}, T \sim 20 \mathrm{~K}\right)$ that occupy only a very small volumetric filling factor within the Local Bubble.

Maness et al. (2009) also propose that disk gas could undergo ram pressure stripping by the ISM, and disk grains are consequently swept away when they become entrained in this outflowing gas. In this scenario, sufficient disk gas is required and the grains must be entrained effectively, neither of which is assured. However, measurements of the NA I doublet towards HD 15115 by Redfield (2007) indicate that the column density for this disk is $\sim 5$ times greater than towards $\beta$ Pic; he estimates that the upper limit on the total gas mass in the circumstellar disk is $\sim 0.3 M_{\oplus}$.

In order for direct ram pressure stripping of disk grains to occur, the force of the ISM on the grains must be comparable to the stellar gravitational force. Yet, Maness et al. (2009) propose that in the case where this condition is not met, neutral gas can still affect disk morphology over timescales of $\sim 10^{3}-10^{4}$ years by introducing secular perturbations to the orbits of bound grains. A similar mechanism has been proposed as the removal mechanism for dust from our Solar System at 20 - 100 AU (Scherer 2000). Like drag from the Solar wind, neutral gas drag involves the transfer of momentum from incident gas particles to grain surfaces. However, unlike the Solar wind, interstellar gas drag tends to increase grain eccentricities and semi-major axes, and can eventually unbind grains from the system. The normalcy of the interstellar densities, velocities, and cloud sizes required by this neutral gas drag model make it a promising mechanism for producing the structures in this family of asymmetrical debris disks. Furthermore, the presence of detectable gas in the disk lends additional credibility to the argument that neutral gas drag may play a role in shaping the disk morphology. 


\subsection{Interactions with Other Perturbers}

An alternative mechanism to ISM interactions that can produce disk asymmetries involves planet induced resonances. Parent planetesimals locked in resonance with orbiting planets can produce large grains that stay in resonance, or, if collisions are unimportant, grains can drift into resonances due to Poynting-Robertson drag (Krivov et al. 2007). Wyatt (2006) predict that in the former case, an asymmetry can be present at short and long wavelengths, but absent at intermediate wavelengths. In this model, the large grains that dominate millimeter emission trace the parent planetesimals and thus exhibit the same clumpy, resonant distribution. The small grains dominant at short wavelengths are preferentially created in the high-density resonant clumps and expelled from the system on short timescales. The mid-sized grains traced by intermediate wavelengths remain bound, but fall out of resonance due to radiation pressure and are scattered into an axisymmetric distribution. Maness et al. (2008) invoke this prediction to explain the multi-wavelength observations of HD 32297, which appears asymmetric at both optical and millimeter wavelengths, but symmetric in the mid-infrared. A similar picture may be hinted at in the case of HD 15115. The scattered light observations are clearly asymmetric, and the SMA observations suggest a potential millimeter asymmetry. However, observations at $3-5 \mu \mathrm{m}$ show a symmetric disk morphology (Rodigas et al. 2012). Detailed dynamical models of these systems are needed to draw firm conclusions about this speculation.

Global disk asymmetries could also be produced by a single or periodic stellar flyby, as proposed to explain the large scale scattered light asymmetry of the $\beta$ Pic debris disk (Kalas \& Jewitt 1995; Kalas et al. 2001). Kalas et al. (2007) point out that the $\beta$ Pic moving group member, HIP 12545, nearby in the sky to HD 15115 (3.9 Moór et al. 2006), could be involved in a past interaction, as these two stars show identical proper motion, galactic space motion, and heliocentric distance within the measured uncertainties. Morever, the present location of HIP 12545 is in the direction of the truncated eastern side of the HD 15115 disk, as in the simulations of (Larwood \& Kalas 2001) flyby interactions. However, there are no indications other than this circumstantial evidence for any interaction. Furthermore, the membership of HD 15115 in the $\beta$ Pic moving group has been called into question. Moór et al. (2006) used kinematic arguments to propose this group membership. The recent Bayesian analysis by Malo et al. (2013) suggests a higher probability of membership in the Columba Association. A better understanding of the group membership and age of HD 15115 certainly would be helpful in unraveling the origins of the debris disk morphology. 


\subsection{Interactions within Disk Material}

Gemini Near-Infrared Coronographic Imager (NICI) data in the H and Ks bands (Mazover et al. 2014) resolved the HD 15115 disk and detected ansae on both sides at a radius of $1.99^{\prime \prime}(\sim 90$ $\mathrm{AU})$. These new observations indicate a ring-like shape with an inner cavity that appears symmetric, in contrast with the east-west brightness asymmetry seen in other images. If the inner ring is truly symmetric, an alternative or additional mechanism for the observed brightness asymmetry is possible. Mazoyer et al. (2014) suggest that both a local increase in the dust grain scattering cross section and/or a local density enhancement of small grains could lead toward an increase in collisions at the location of the western brightness peak. A similar explanation was recently invoked to explain the millimeter emission clump seen in ALMA observations of the $\beta$ Pictoris disk (Dent et al. 2014).

We can crudely estimate the mass of small grains needed to account entirely for the tentative excess millimeter emission on the western side of the disk. Optically thin dust emission at a temperature, $T_{\text {dust }}$, has flux $F_{\text {dust }} \approx \kappa_{\nu} B_{\nu}\left(T_{\text {dust }}\right) M_{\text {dust }} / d^{2}$, where $\kappa_{\nu}$ is the dust opacity, $B_{\nu}$ is the Planck function, $M_{\text {dust }}$ is the dust mass, and $d=45 \mathrm{pc}$. We estimate a characteristic temperature for dust in this excess feature of $\sim 30 \mathrm{~K}$, using its approximate radial location ( $\sim 160 \mathrm{AU})$ and assuming radiative equilibrium of starlight with blackbody grains (Backman \& Paresce 1993). To estimate an appropriate dust opacity, we assume that the grain size distribution is described by a power-law between $0.1 \mu \mathrm{m}$ and some maximum size $a_{\max }, n(a) \propto a^{q}$. For grains with composition volume fractions of $0.07,0.21,0.42$, and 0.30 for silicate, carbon, ice, and vacuum, respectively, Ricci et al. (2010) calculate dust opacities as a function of $a_{\max }$. (Note, the results are not very sensitive to other reasonable assumptions about composition.) Adopting a typical power-law exponent $q=$ -3.5 , and maximum grain size $a_{\max }=0.1$ millimeter, models yield a dust opacity, $\kappa_{\nu} \sim 3$ $\mathrm{cm}^{2} \mathrm{~g}^{-1}$. Given these assumptions, the mass of small grains required to account for the excess millimeter flux is $\sim 1 \times 10^{26} \mathrm{~g}$, or $\sim 1.4$ times the lunar mass. As this large mass indicates, a very significant collision would be required for small grains to account entirely for the western millimeter emission feature. Furthermore, such a collisional feature is transient and can persist for only a small fraction of the system age (Jackson et al. 2014).

Lyra \& Kuchner (2013) discuss an additional mechanism by which disk gas can generate azimuthal asymmetries through the "photoelectric instability" effect. In this scenario, a dense region of dust heats the gas through photoelectric heating and creates a local pressure maximum, which in turn attracts additional dust. The resulting instability could lead to the development of rings, spirals, or other structures within the disk. Lyra \& Kuchner (2013) suggest that this effect will occur in disks with a total disk dust to gas mass ratio, $\epsilon \sim 1$. In the case of HD 15115, estimates of the total dust and gas mass in the disk suggest that 
$\epsilon>0.16$, in the regime where this mechanism could work. However, without further analysis, it is unclear whether the level of azimuthal asymmetries produced would be large enough to account for these new observations.

In short, there is still no clear consensus as to which of these mechanisms is responsible for shaping the debris around HD 15115 and other, similar systems (notably HD 61005 and HD 32297). Each mechanism affects different sized grains preferentially and operates under specific physical conditions. Improved constraints on the detailed morphologies of these systems are required to reach definitive conclusions.

\section{Conclusions}

We present SMA 1.3 millimeter observations that resolve the emission from the HD 15115 debris disk, the first at such a long wavelength. The bulk of the millimeter emission is described well by a symmetric belt, consistent with the presence of a "birth ring" of collisional planetesimals indicated by previous observations of scattered light. The SMA observations also show $\mathrm{a} \sim 3 \sigma$ residual feature coincident with the western extension of the scattered light along the disk major axis. If real, this additional feature hints that the distribution of larger grains may be asymmetric as well.

There is no consensus concerning the physical mechanism or mechanisms responsible for the scattered light asymmetries in HD 15115 and other, similar debris disks. Ram pressure from surrounding interstellar gas can strip both bound and unbound grains, or induce outflows of disk gas that entrain grains. However, the tentative millimeter emission asymmetry disfavors any process that affects only small grains. An alternative mechanism invokes neutral gas in the disk that introduces secular perturbations to grain orbits, producing significant effects on disk morphology on orbital timescales (Maness et al. 2009). The presence of atomic gas towards HD 15115 suggests that this idea should be explored more thoroughly. Interactions with perturbers are another possibility. Dust-trapping resonances from orbiting planets may be able to explain the observed wavelength-dependent structure. Future observations of HD 15115 at higher angular resolution and sensitivity with the Atacama Large Millimeter/Submillimeter Array are needed to resolve the detailed morphology of the millimeter emission and to address the processes responsible for shaping this remarkable debris disk.

We thank Paul Kalas for providing the Hubble Space Telescope image in Figure 1. M.A.M acknowledges support from a National Science Foundation Graduate Research Fellowship (DGE1144152). We thank Katherine Rosenfeld, Til Birnstiel and John Debes for 
helpful conversations.

\section{REFERENCES}

Augereau, J.-C., \& Beust, H. 2006, A\&A, 455, 987

Backman, D. E., \& Paresce, F. 1993, in Protostars and Planets III, ed. E. H. Levy \& J. I. Lunine, 1253-1304

Binks, A. S., \& Jeffries, R. D. 2014, MNRAS, 438, L11

Boccaletti, A., et al. 2012, A\&A, 544, A85

Burns, J. A., Lamy, P. L., \& Soter, S. 1979, Icarus, 40, 1

Currie, T., et al. 2012, ApJ, 757, 28

Debes, J. H., Weinberger, A. J., \& Kuchner, M. J. 2009, ApJ, 702, 318

Debes, J. H., Weinberger, A. J., \& Song, I. 2008, ApJ, 684, L41

Dent, W. R. F., et al. 2014, Science, 343, 1490

Foreman-Mackey, D., Hogg, D. W., Lang, D., \& Goodman, J. 2013, PASP, 125, 306

Gáspár, A., Psaltis, D., Özel, F., Rieke, G. H., \& Cooney, A. 2012, ApJ, 749, 14

Goodman, J., \& Weare, J. 2010, Commun. Appl. Math. Comput. Sci., 5, 65

Hines, D. C., et al. 2007, ApJ, 671, L165

Ho, P. T. P., Moran, J. M., \& Lo, K. Y. 2004, ApJ, 616, L1

Jackson, A. P., Wyatt, M. C., Bonsor, A., \& Veras, D. 2014, MNRAS, 440, 3757

Kalas, P., Deltorn, J.-M., \& Larwood, J. 2001, ApJ, 553, 410

Kalas, P., Fitzgerald, M. P., \& Graham, J. R. 2007, ApJ, 661, L85

Kalas, P., \& Jewitt, D. 1995, AJ, 110, 794

Krivov, A. V., Queck, M., Löhne, T., \& Sremčević, M. 2007, A\&A, 462, 199

Larwood, J. D., \& Kalas, P. G. 2001, MNRAS, 323, 402 
Lebreton, J., et al. 2012, A\&A, 539, A17

Lyra, W., \& Kuchner, M. 2013, Nature, 499, 184

MacGregor, M. A., et al. 2013, ApJ, 762, L21

Malo, L., Doyon, R., Lafrenière, D., Artigau, É., Gagné, J., Baron, F., \& Riedel, A. 2013, ApJ, 762, 88

Maness, H. L., Fitzgerald, M. P., Paladini, R., Kalas, P., Duchene, G., \& Graham, J. R. 2008, ApJ, 686, L25

Maness, H. L., et al. 2009, ApJ, 707, 1098

Marzari, F., \& Thébault, P. 2011, MNRAS, 416, 1890

Matthews, B. C., Krivov, A. V., Wyatt, M. C., Bryden, G., \& Eiroa, C. 2014, ArXiv e-prints

Mazoyer, J., Boccaletti, A., Augereau, J.-C., Lagrange, A.-M., Galicher, R., \& Baudoz, P. 2014, ArXiv e-prints

Moór, A., Ábrahám, P., Derekas, A., Kiss, C., Kiss, L. L., Apai, D., Grady, C., \& Henning, T. 2006, ApJ, 644, 525

Moór, A., et al. 2011, ApJS, 193, 4

Mundy, L. G., et al. 1996, ApJ, 464, L169

Panić, O., et al. 2013, MNRAS, 435, 1037

Redfield, S. 2007, ApJ, 656, L97

Ricarte, A., Moldvai, N., Hughes, A. M., Duchêne, G., Williams, J. P., Andrews, S. M., \& Wilner, D. J. 2013, ApJ, 774, 80

Ricci, L., Testi, L., Natta, A., Neri, R., Cabrit, S., \& Herczeg, G. J. 2010, A\&A, 512, A15

Rodigas, T. J., et al. 2012, ApJ, 752, 57

Scherer, K. 2000, J. Geophys. Res., 105, 10329

Schneider, G., et al. 2014, ArXiv e-prints

Silverstone, M. D. 2000, PhD thesis, UNIVERSITY OF CALIFORNIA, LOS ANGELES

Strubbe, L. E., \& Chiang, E. I. 2006, ApJ, 648, 652 
van Leeuwen, F. 2007, A\&A, 474, 653

Williams, J. P., \& Andrews, S. M. 2006, ApJ, 653, 1480

Wilner, D. J., Andrews, S. M., \& Hughes, A. M. 2011, ApJ, 727, L42

Wilner, D. J., Andrews, S. M., MacGregor, M. A., \& Hughes, A. M. 2012, ApJ, 749, L27

Wyatt, M. C. 2006, ApJ, 639, 1153 
Table 1. Submillimeter Array Observations of HD 15115

\begin{tabular}{ccccccc}
\hline \hline $\begin{array}{c}\text { Observation } \\
\text { Date }\end{array}$ & $\begin{array}{c}\text { Array } \\
\text { Config. }\end{array}$ & $\begin{array}{c}\text { Number of } \\
\text { Antennas }\end{array}$ & $\begin{array}{c}\text { Baseline } \\
\text { Lengths }(\mathrm{m})\end{array}$ & $\begin{array}{c}\text { LO Freq. } \\
(\mathrm{GHz})\end{array}$ & $\begin{array}{c}\text { HA } \\
\text { Range }\end{array}$ & $\begin{array}{c}225 \mathrm{GHz} \text { atm. } \\
\text { Opacity }^{\mathrm{a}}\end{array}$ \\
\hline 2013 Sep 15 & Extended & 6 & $25-174$ & 225.5 & $-4.2,3.3$ & 0.08 \\
2013 Oct 19 & Extended & 5 & $20-182$ & 235.5 & $-3.8,4.9$ & 0.28 \\
2013 Nov 18 & Extended & 6 & $23-174$ & 225.5 & $-2.3,3.1$ & 0.10 \\
2013 Dec 10 & Compact & 6 & $8-53$ & 225.5 & $-1.3,3.7$ & 0.20 \\
2013 Dec 12 & Compact & 6 & $6-53$ & 224.9 & $-4.5,4.8$ & 0.11 \\
\hline
\end{tabular}

Note. - ${ }^{\mathrm{a}}$ characteristic value for the track measured at the nearby Caltech Submillimeter Observatory

Table 2. Model Parameters

\begin{tabular}{llcc}
\hline \hline Parameter & \multicolumn{1}{c}{ Description } & Best-Fit & 68\% Confidence Interval \\
\hline$F_{\text {belt }}$ & Belt flux density (mJy) & 2.56 & $+0.50,-0.83$ \\
$R_{\text {in }}$ & Belt inner radius (AU) & 43.4 & $+28.3,-28.3$ \\
$R_{\text {out }}$ & Belt outer radius (AU) & 113. & $+30.6,-21.8$ \\
$x$ & Belt radial power law index & -0.75 & $+1.37,-0.87$ \\
\hline$F_{\text {res }}$ & Point source flux (mJy) & 0.84 & $+0.16,-0.12$ \\
$\Delta r$ & Point source offset (") & -3.55 & $+0.45,-0.12$ \\
\hline$\Delta \alpha$ & R.A. offset of belt center $\left(^{\prime \prime}\right)$ & 1.26 & $+0.07,-0.05$ \\
$\Delta \delta$ & Decl. offset of belt center $\left(^{\prime \prime}\right)$ & -0.78 & $+0.09,-0.05$ \\
\hline
\end{tabular}




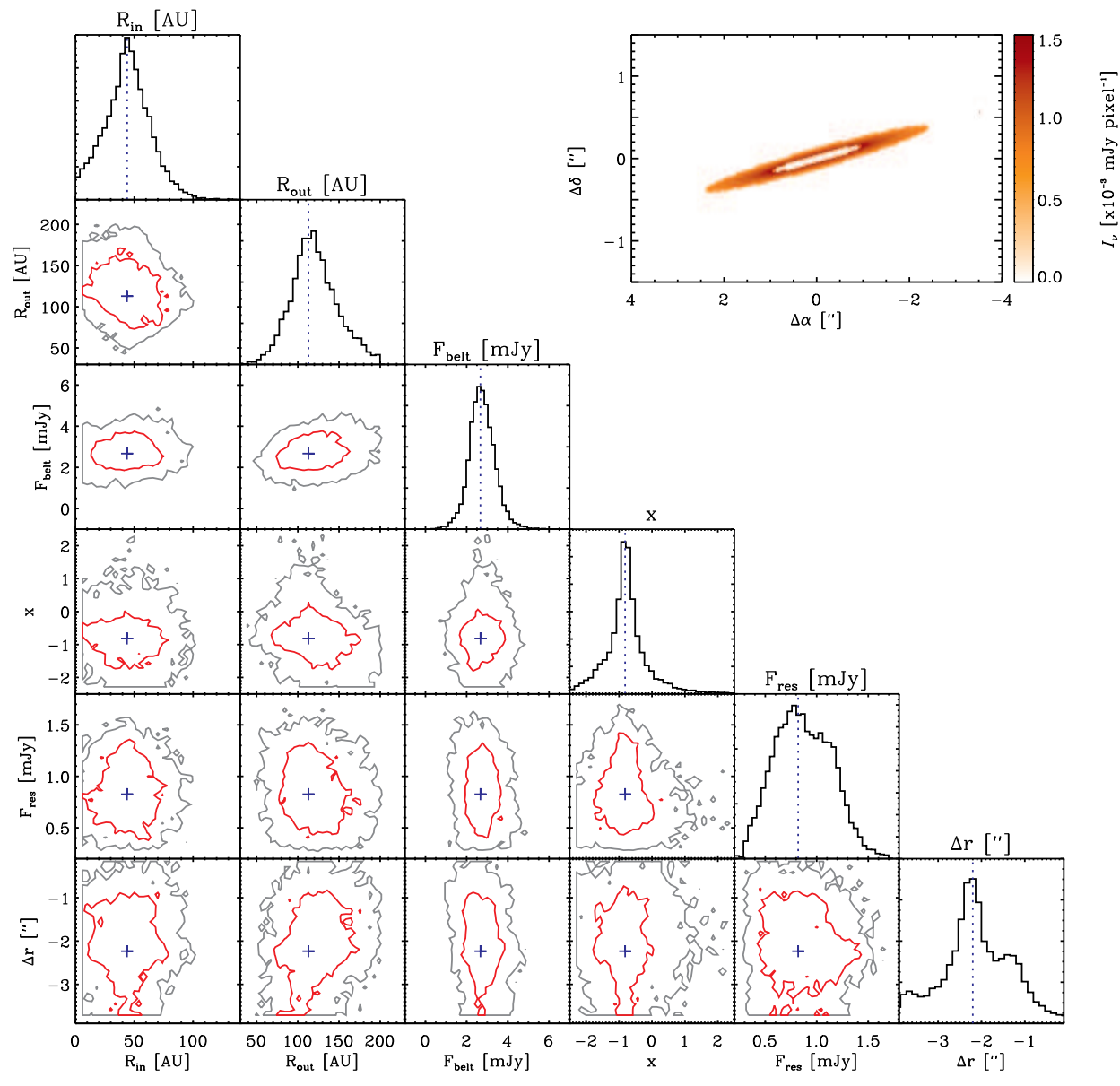

Fig. 2. - A sample of the output from a run of $\sim 10^{4}$ MCMC trials for the 6 best-fit model belt parameters $\left(R_{\text {in }}, R_{\text {out }}, F_{\text {belt }}, x, F_{\text {res }}\right.$, and $\left.\Delta r\right)$. The diagonal panels show the 1D histogram for each parameter marginalized over all other parameters considered in the model. For each parameter, the peak of each histogram is taken to be the best-fit value. The remaining panels show contour plots of the $1 \sigma$ (red) and $2 \sigma$ (gray) regions for each pair of parameters, with the blue crosses marking the best-fit values. The inset panel in the upper right shows the resulting best-fit model at full resolution, pixel scale $\sim 0$ ' 02 (0.9 AU). The $1 \sigma$ and $2 \sigma$ regions are determined by assuming normally distributed errors, where the probability that a measurement has a distance less than $a$ from the mean value is given by $\operatorname{erf}\left(\frac{a}{\sigma \sqrt{2}}\right)$. 

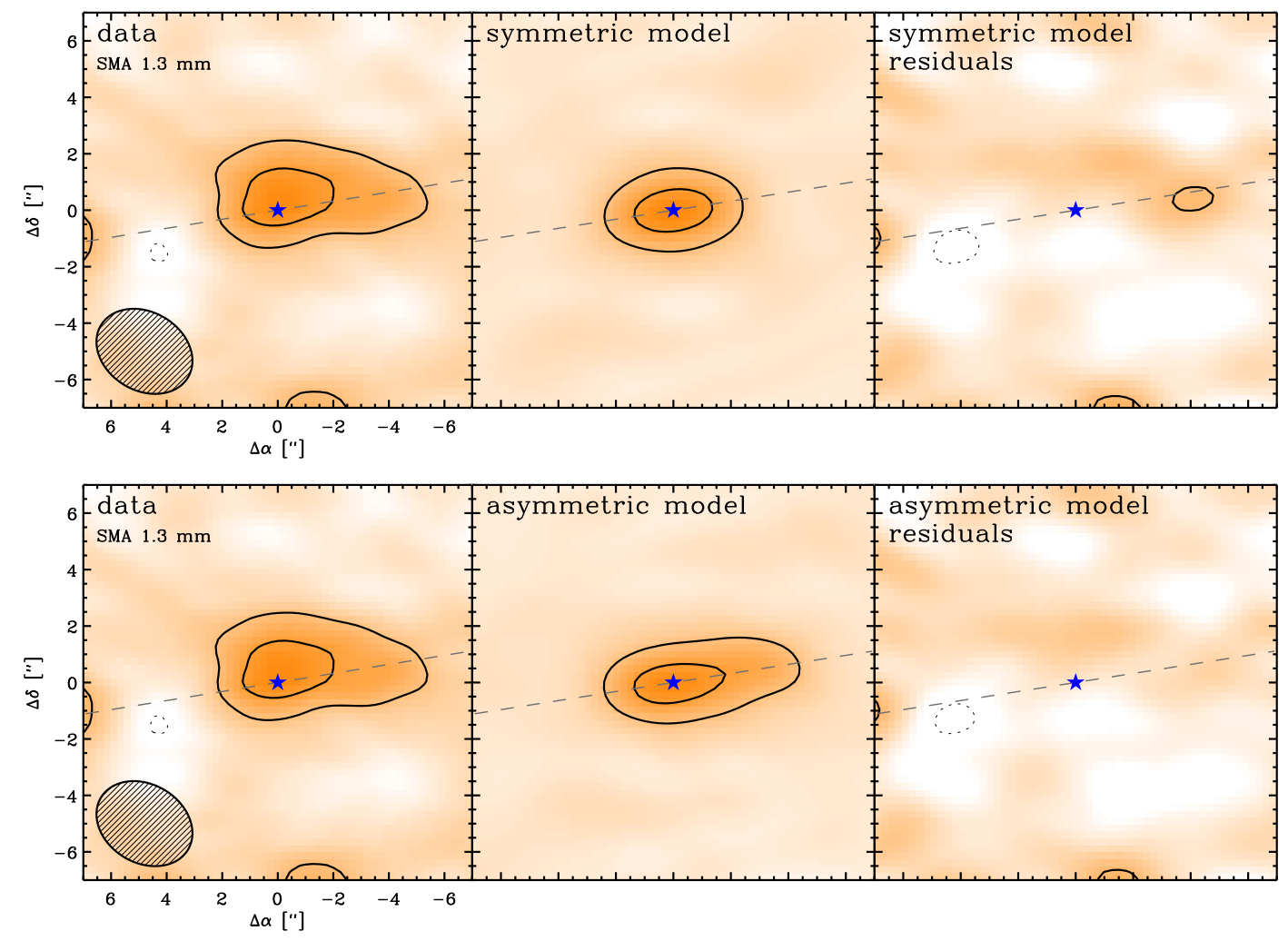

Fig. 3.- (upper): (left) The 1.3 millimeter continuum emission from HD 15115 observed with the SMA, as in Figure1, (center) image of the best-fit azimuthally symmetric disk model (see Section 3 for a description of the modeling formalism and results), and (right) the imaged residuals from the symmetric model, showing a $3 \sigma$ feature on the western side of the disk. (lower): (left) the SMA image, (center) image of the best-fit asymmetric model, and (right) the imaged residuals from the asymmetric model, which do not show any remaining significant features. The contour levels are at $2 \sigma\left(0.6 \mathrm{mJy}^{\text {beam }}{ }^{-1}\right)$ intervals in all panels. 